\title{
Evaluation of 3D Model Segmentation Techniques based on Animal Anatomy
}

\author{
Nasim Hajari, Irene Cheng, Anup Basu \\ Department of Computing Science \\ University of Alberta \\ Canada
}

\begin{abstract}
D model decomposition is a challenging and important problem in computer graphics. Several semantically based approaches have been proposed in the literature; however, due to the lack of proper evaluation criteria, comparison of these techniques is almost impossible. In this paper we suggest to use animal anatomy as the ground truth and compare the result of different segmentation techniques based on that. Differing from previous approaches which perform the evaluation based on ground truth databases created subjectively by human observers, we consider expert knowledge on anatomy of various animals. Based on this knowledge we specify the ground truth for different animals and compare alternative algorithms.
\end{abstract}

\section{Keywords-3D segmentation, skeletonization, anatomy}

\section{INTRODUCTION}

One of the most challenging and important problems in computer graphics, which has recently attracted many researchers' attention, is 3D model decomposition or mesh segmentation. The problem is segmenting a mesh or volume into components based on some geometric or semantic criteria. Mesh segmentation has several applications in modeling, texture mapping, animation, compression, simplification and so on.

There are two different approaches to 3D mesh segmentation [1]. It can be done either geometrically or semantically. In geometric mesh segmentation the mesh is segmented into a number of visually meaningless patches based on some surface properties such as curvature, distance to a fitting plane or surface normal. However, in a semantic approach, the mesh is segmented into visually meaningful parts that are related to relevant features of the shape. For instance one can segment the 3D model of a horse into head, body, legs and tail. The semantic approach attracts more attention because of its strength in different applications, such as animation, classification, shape retrieval and skeleton extraction. The main challenge with this approach is the lack of a consistent evaluation criteria to compare different techniques, since each technique has been developed for a specific application. Therefore, the comparison of different segmentation techniques should be based on the application. Another problem is the definition of meaningful patches for 3D models. For example, one can divide an animal body, such as a horse, into coarse segments, head, body, legs and tail.

\author{
Guillaume Lavoué \\ Université de Lyon, CNRS \\ Insa-Lyon, LIRIS UMR 5205 \\ France
}

While others may segment it based on more detailed animal anatomy; thus, the quantitative comparison can be challenging.

Semantically oriented segmentation of quadrupeds is highly relevant for object retrieval and animation. However, because of the abovementioned reasons there is no obvious metric to evaluate the accuracy and quality of segmentation results. Several previous works have been proposed for segmentation evaluation [2], [3]. They rely on databases of human segmentation of 3D models. However, we argue that arbitrary human observers do not necessarily provide a correct semantic ground-truth to evaluate the segmentation. In this paper the similarity between segmentation results and an animal anatomy is considered as the evaluation metric.

In this work we also extend an anatomy preserving segmentation technique. The underlying idea is using the skeleton of a 3D model to guide the decomposition. The skeleton of a 3D model is a compact graph-like abstraction derived from the centerlines of the original model [4]. The next step is mapping surface points onto skeleton branches. Finally, each set of surface points that map onto the same skeleton branch is considered as one patch. This approach uses both global shape and local features, helping enhance perceptual quality, which is required in animation and game type applications. In prior work, [4] used only one anatomy model (a horse) as the ground truth and evaluated the segmentation results only for the horse model. This is not adequate for any general findings. In this paper, we segment other 3D animal models and compare the results with animal anatomy to evaluate various algorithms.

As mentioned above, ground truth for evaluation of segmentation results has been typically obtained solely through human input [2], [3], [5] in the computer graphics and vision communities. However, this approach is purely subjective and could vary significantly from one user group to another. The importance of accurate and consistent segmentation has been pointed out by researchers in various application communities. For example, why defining ground truth for tables [6] is difficult has been discussed in [7]. Defining ground truth for various fields in medicine has been extensively researched, e.g., [8]. Validation of the segmentation and expert definitions of ground truth has also been carefully measured [9]. By contrast, the expectations of the quality of ground truth in vision and graphics research do 
not seem to be that high. For example, in Figure 1 in [5] the elephants are identified as one object in one segmentation, while three in others. Despite issues like this, the different segmentations are considered to be "highly consistent." It appears that application domains, and what level of accuracy and consistency are necessary in them, have been largely ignored in vision and graphics research. Thus, we propose using biologically defined anatomical models as a more reliable approach to ground truth definition and subsequent evaluation for a class of objects in 3D segmentation.

The remainder of this paper is organized as follows: Section II discusses some of the semantic segmentation techniques. Section III explains our skeleton based segmentation approach. In Section IV the evaluation criteria and the proposed anatomical ground-truth are presented. Section V compares the results of skeleton based segmentation with the most efficient state of the art methods. Concluding remarks are given in Section VI.

\section{RELATED WORK}

Semantically oriented segmentation techniques try to generate the segmentation patches such that a cost function is minimized based on a given criterion. The main difference among various algorithms in this category is the cost function and the criterion used. In this section we will explain some of these techniques in greater detail.

The authors in [10] proposed a hierarchical decomposition technique using fuzzy clustering. This algorithm is based on a hierarchical tree. Each node in the tree is associated with the mesh of a particular patch and the root is associated with the whole input object. The higher level nodes correspond to coarser patches while leaves and lower level nodes correspond to finer patches. The algorithm determines a suitable number (k) of patches at each node, and then computes a k-way segmentation of this node. First, the algorithm finds the meaningful components along with the boundaries between the components that are considered to be fuzzy. The next step is finding the exact boundaries in the fuzzy areas which preserve the features of the object. However, the approach relaxes the condition that every face should belong to exactly one patch and allows fuzzy membership, which means that each face has probabilities associated to belonging to different patches. This probability is based on geodesic and angular distances between all pairs of the faces. Another hierarchical method has been proposed in [11]. This algorithm is also based on a hierarchical tree and proceeds from coarse to fine scale. The main advantage of this technique is that it is insensitive to pose and proportions. The approach first transforms the mesh vertices into a pose invariant space, then robustly extracts the feature points, and finally extracts the core component of the mesh. [12] proposed an algorithm based on the fitting primitives. Initially, each triangle of a triangular mesh corresponds to a single cluster. All the pairs of adjacent clusters are considered and compared at every iteration, and the one that can be best approximated by one of the primitives forms a new single cluster. The primitives are planes, spheres and cylinders; and, an L2 metric is used to compare each combination of the cluster with one of these primitives.
The Shape Diameter Function (SDF) is another 3D segmentation approach which has been proposed in [13]. The SDF is defined as the diameter of the object in the neighborhood of each point on its surface. Given a point on the surface mesh a set of rays is sent inside a cone centered around its inward-normal direction (the opposite direction of its normal) to the other side of the mesh. The value of the SDF at the point is defined as the weighted average of all the lengths of rays that fall within one standard deviation from the median of all lengths. [14] presented a segmentation technique based on random cuts. The idea is to generate a random set of mesh segmentations, and then measure how often each edge of the mesh lies on a segmentation boundary in the randomized set. An interactive approach based on random walk has been proposed in [15]. The user chooses some faces as seeds, then a probability is assigned to each of the three edges of non-seed faces. This value determines whether or not a random walker moves across a particular edge to the corresponding face. A face belonging to the region is grouped with the seed $\mathrm{X}$ if a random walker starting at that face has a higher probability of reaching the seed $X$ than any other seeds.

\section{SEGMENTATION BASED ON SKELETONIZATION}

Since the human eyes are very sensitive to changes along the boundary of an object it is logical to use the structural shape for simplification and segmentation purposes. Since the projected $2 \mathrm{D}$ contour of a $3 \mathrm{D}$ model can vary significantly depending on a change of view, it is more effective to use the model skeleton in order to guide segmentation. The skeleton of a 3D model is a compact graph-like abstraction derived from the centerlines of the original model [4].

It is very important to extract the skeleton as accurately as possible. For segmentation purposes a unit width skeleton, such as the Valance Normalized Spatial Median (VNSM) [16] is needed. However, these skeletons are very prone to local and global noise and may contain some unwanted branches. The Scale Space and Gaussian filters can be used to remove the noise and smooth the skeleton. The unwanted branches should be removed considering the length and topological position of a branch [4].

After extracting the smooth and noise-free skeleton, the next step is to decompose the model into different segments according to the following steps.

- Decompose the skeleton at junction points. Junction points are the points that are connected to more than one skeleton branch.

- Map surface nodes to skeleton branches. This is based on the L2 distance between surface nodes and skeleton vertices. In other words, to map a point on the surface to a skeleton branch node, it should be close to the skeleton branch nodes, with skeleton-tosurface normal vector pointing in a direction similar to the surface normal vectors. Also, the neighboring 
nodes should have the same label if the curvature is close. These distances are computed for each surface point or a subset of surface points against all skeleton vertices.

- The last step is labeling the neighborhood nodes. The previous step just labeled some selected sample points. The watershed method is used to flood the labels from samples.

\section{EVALUATION}

The main challenge for semantically oriented segmentation is defining the evaluation criteria, which is related to the application. However, for animation purposes and generating natural movements of a model, a more meaningful segmentation for animals is based on anatomy. Shi et al. [4] used a metric function distance for evaluation purposes. The error is then the Euclidean distance between registered pair of cuts plus the length of non-paired cuts. However, they just used one anatomy model, that of a horse, as the ground truth and evaluated the segmentation results only for the horse model. This limited evaluation is not adequate.

For evaluation purposes we used the same method as Shi et al. First, we registered different cuts to the corresponding ground truth ones; then calculated the Euclidean distance for each cut plus the length of non-paired cuts. Smaller errors imply better automatic segmentation compared to the ground truth. Since the ground truth is generated manually based on animal anatomies, different experts may specify slightly different ground truths. However, this does not affect the comparison results very much, since the ground truth is not solely based on human perception but anatomical information is also considered in the process.

We use sheep [17], cow [17], dog [18] and giraffe [19] anatomies. There are several free 3D models of these animals available on public databases [3], [20]. These anatomy models are very detailed and in order to use them as the ground truth, we manually segment them at a coarse level. However, it is possible to ask the user what level of details he/she prefers. Figure 1 shows the anatomy models for these animals. Figure 2 shows the corresponding ground-truth segmentations. Figure 3 presents the ground-truth of giraffe at a finer level of detail.

\section{RESUlts}

Using the method proposed in [4], we extracted the skeletons and performed 3D segmentation for cow, sheep, dog and giraffe. Figure 4 shows the extracted skeletons. Figure 5 compares the segmentation results with random walk (RW) [15], random cuts (RC) [14], shape diameter (SDF) [13] and the ground truth. Note that the extracted skeletons may still contain some noise or unwanted branches which can lead to poor segmentation quality. One technique that can be used to eliminate the noise and improve the generated skeletons is scale space filtering. In future work we will use this method and other techniques to improve the results. Table I shows the metric distance between the segmented models and the corresponding animal anatomies. It is based on manual

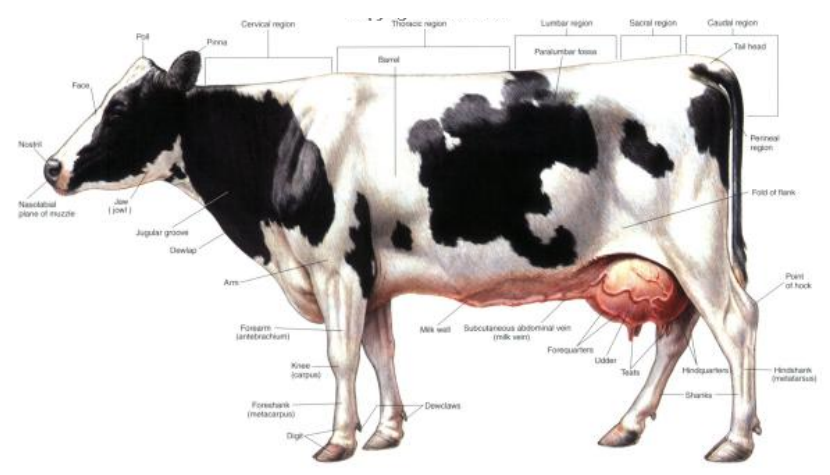

(a)

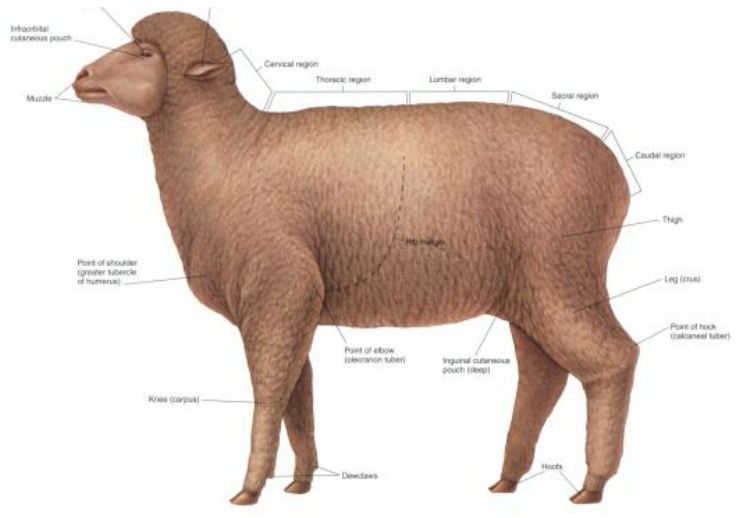

(b)

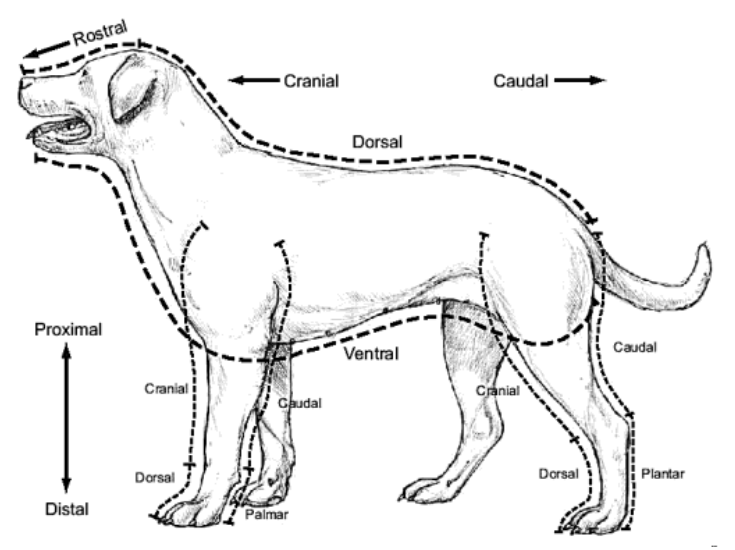

(c)

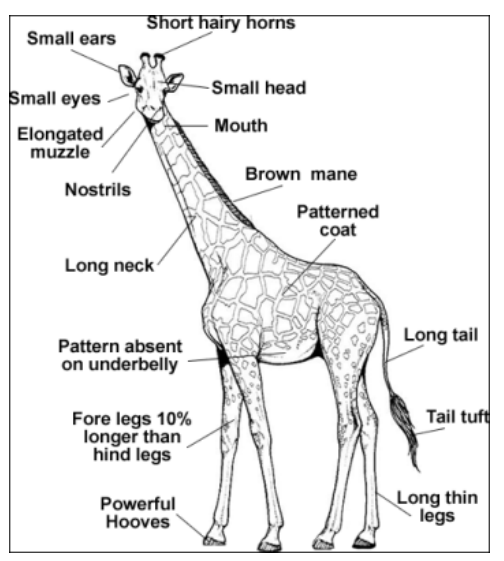

(d)

Fig. 1. The anatomy model of (a) cow [17], (b) sheep [17], (c) dog [18] and (d) giraffe . 


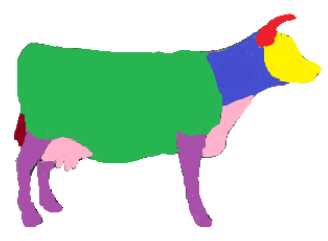

(a)

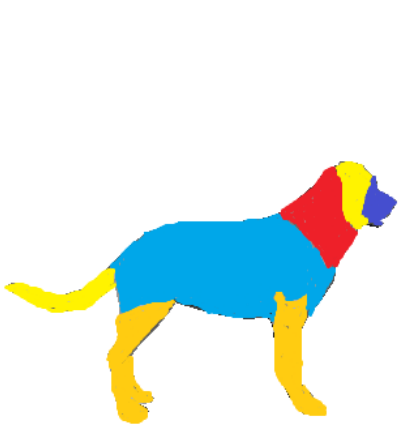

(c)

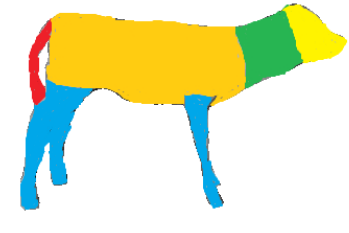

(b)

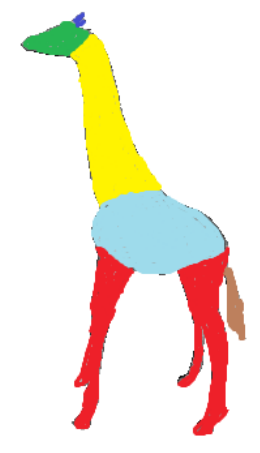

(d)
Fig. 2. The manual segmentation for (a) cow, (b) sheep, (c) dog and (d) giraffe based on their anatomy.

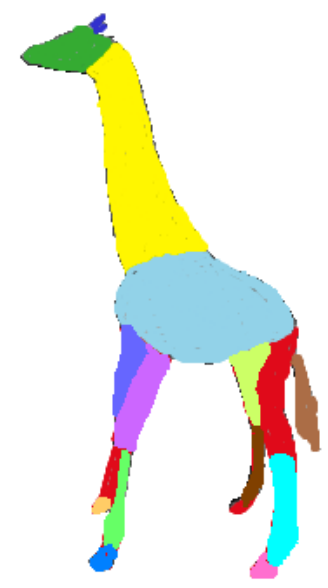

Fig. 3. Detailed segmentation for the giraffe.

registration of the animal anatomy and generated segments and calculating the Euclidean distance for each segment, plus any extra segments that the methods generated. Our method as well as SDF [13] produce the best results. It is interesting to notice that with our anatomically defined ground truth, SDF [13] is better than random cuts [14]; this contradicts the evaluation based on the Princeton segmentation benchmark [3], which is entirely human defined. This confirms that human segmentations may not necessarily be a good means of defining ground truth for some applications.

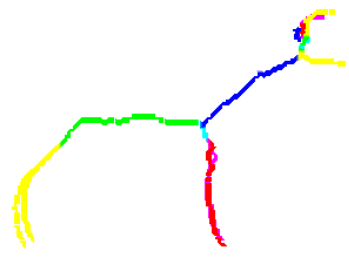

(a)

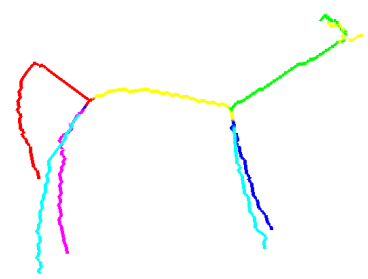

(b)

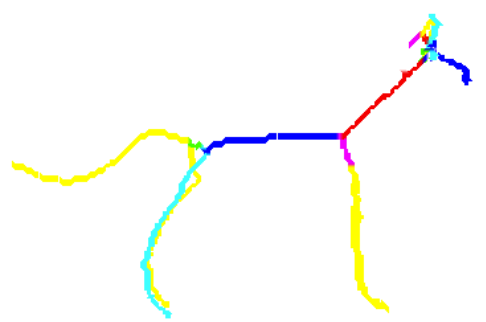

(c)

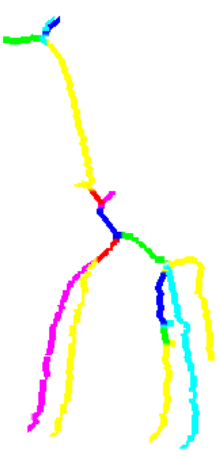

(d)
Fig. 4. Extraced skeletons for (a) cow, (b) sheep, (c) dog and (d) giraffe. Different colors are used for different segments of the skeleton.

\begin{tabular}{|l|l|l|l|l|}
\hline & RW & RC & SDF & Skeleton \\
\hline Cow & 0.77 & 0.71 & 0.65 & 0.57 \\
\hline Horse & 1.00 & 0.84 & 0.79 & 0.45 \\
\hline Dog & 0.8 & 0.75 & 0.25 & 0.65 \\
\hline Giraffe & 0.64 & 0.43 & 0.57 & Too noisy \\
\hline Detailed giraffe & 0.48 & 0.58 & 0.28 & 0.79 \\
\hline
\end{tabular}

TABLE I. METRIC DISTANCE BETWEEN DIFFERENT SEGMENTATION METHODS AND GROUNDTRUTH.

\section{CONCLUSION}

We described an approach to obtaining ground truth for animal model segmentation based on expert knowledge, and used this information to compare various approaches. In future work, we will extend our study using a larger collection of animals. We will consider extracting the ground truth at different levels of detail. Furthermore, we will study how current algorithms can be extended to segment at varying levels of detail. One modification that can be done in the future is to let the user define the level of detail, or at least let the program know if the user needs a more detailed or a coarse segmentation. Then, a program can generate the skeleton and segmentation according to the user requirements.

\section{REFERENCES}

[1] M. Attene, S. Katz, M. Mortara, G. Patane, M. Spagnuolo, and A. Tal, "Mesh segmentation - a comparative study," in IEEE International Conference on Shape Modeling and Applications. 2006, SMI '06, pp. 7-, IEEE Computer Society.

[2] H. Benhabiles, J-P. Vandeborre, G. Lavoue, and M. Daoudi, "A framework for the objective evaluation of $3 \mathrm{~d}$ models using a ground truth of human segmented 3d models," in IEEE Shape Modeling and Applications, 2009.

[3] X. Chen, A. Golovinskiy, and T. Funkhouser, "A benchmark for 3d mesh segmentation," ACM Trans. Graph., vol. 28, pp. 73:1-73:12, 2009. 


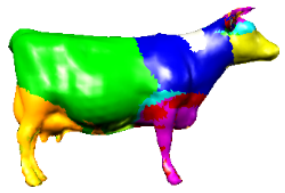

(a)

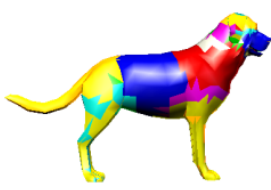

(f)

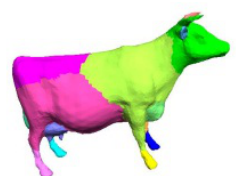

(b)

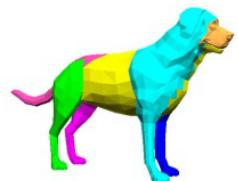

$(\mathrm{g})$

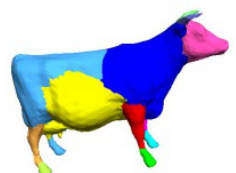

(c)

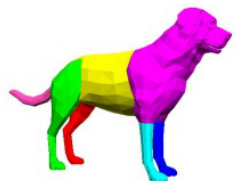

(h)

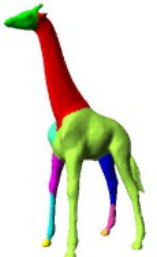

(m)

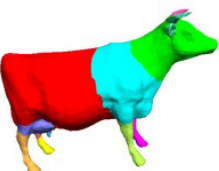

(d)

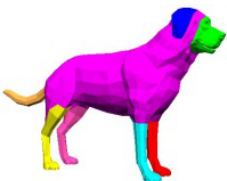

(i)

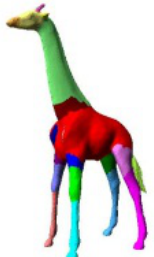

(n)

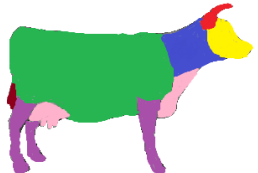

(e)

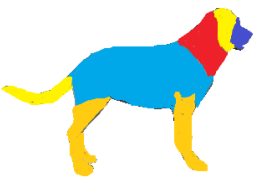

(j)

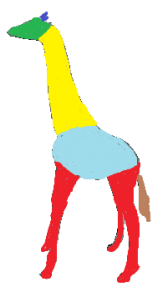

(o)

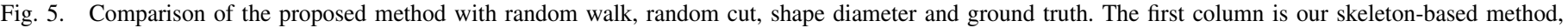

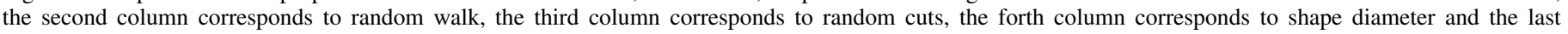
column is the ground truth. Top row shows segmentation for the cow, middle for the dog, and bottom for the giraffe.

[4] L. Shi, I. Cheng, and A. Basu, "Anatomy preserving 3d model decomposition based on robust skeleton-surface node correspondence," in IEEE International Conference on Multimedia and Expo, 2011, pp. $1-6$.

[5] David Martin, Charless Fowlkes, Doron Tal, and Jitendra Malik, "A database of human segmented natural images and its application to evaluating segmentation algorithms and measuring ecological statistics,' in International Conference on Computer Vision, 2001.

[6] B. A. Yanikoglu and L. Vicent, "Pink panther: A complete environment for ground-truthing and benchmarking document page segmentation," Pattern Recognition, vol. 31, no. 9, pp. 1191-1204, 1998.

[7] J. Hu, R. Kashi, D. Lopresti, G. Nagy, and G. Wilfong, "Why table ground-truthing is hard," in International Conference on Document Analysis and Recognition, 2001.

[8] M. Prastawa, E. Bullitt, and G. Gerig, "Synthetic ground truth for validation of brain tumor mri segmentation," in MICCAI, 2005, pp. 26-33.

[9] S. Warfield, K.H. Zou, and W.M. Wells, "Validation of image segmentation and expert quality with an expectation maximization algorithm,' in MICCAI, 2002, pp. 298-306.

[10] S. Katz and A. Tal, "Hierarchical mesh decomposition using fuzzy clustering and cuts," ACM Trans. Graph., vol. 22, no. 3, pp. 954-961, July 2003.

[11] S. Katz, G. Leifman, and A. Tal, "Mesh segmentation using feature point and core extraction," The Visual Computer, vol. 21, no. 8-10, pp. 649-658, 2005.

[12] M. Attene, B. Falcidieno, and M. Spagnuolo, "Hierarchical mesh segmentation based on fitting primitives," Vis. Comput., vol. 22, no. 3, pp. 181-193, Mar. 2006.

[13] L. Shapira, A. Shamir, and D. Cohen-Or, "Consistent mesh partitioning and skeletonisation using the shape diameter function," Vis. Comput., vol. 24, no. 4, pp. 249-259, Mar. 2008.

[14] Aleksey A. Golovinskiy and T. Funkhouser, "Randomized cuts for 3d mesh analysis," ACM Trans. Graph., vol. 27, no. 5, pp. 145:1-145:12, Dec. 2008.

[15] Y.-K. Lai, S.-M. Hu, R.R. Martin, and P.L. Rosin, "Fast mesh segmentation using random walks," in ACM symposium on Solid and physical modeling, 2008, pp. 183-191.

[16] T. Wang, I. Cheng, V. Lopez, E. Bribiesca, and A. Basu, "Valence normalized spatial median for skeletonization and matching," in ICCV Workshops, 2009, pp. 55-62.

[17] T.O. McCracken, R.A. Kainer, T.L. Spurgeon, and G. Brooks, Spurgeon's Color Atlas of Large Animal Anatomy: The Essentials, John Wiley and Sons Ltd, 2008.

[18] T.O. McCracken, R.A. Kainer, and D. Carlson, Color Atlas of Small Animal Anatomy the essentials, Iowa State University Press, 2009.

[19] ," http://www.animalcorner.co.uk/wildlife/giraffes/giraffe_anatomy. html.

[20] P. Shilane, P. Min, M. Kazhdan, and T. Funkhouser, "The princeton shape benchmark," in Shape Modeling International, 2004, pp. 167178. 